\title{
LA PSICOLOGÍA POSITIVA: SU RELACIÓN CON LA SALUD Y EL BIENESTAR
}

\author{
POSITIVE PSYCHOLOGY: ITS RELATION WITH HEALTH \\ AND WELL-BEING
}

\author{
Marcela Sánchez Estrada'
}

RESUMEN

La psicología positiva ha tenido un importante desarrollo en las últimas décadas y hay antecedentes de estudios de aspectos positivos desde mediados del siglo pasado, pero no es hasta los años 90 cuando se observa su desarrollo. Inicialmente propuso cambiar la visión tradicional de tratamiento y curación hacia la de salud y bienestar, y actualmente su estudio se dirige hacia el funcionamiento humano óptimo a partir de fortalezas, virtudes, condiciones y procesos para identificar y promover los factores que permitan prosperar a las personas y comunidades, lo cual implica no solo un enfoque individual, sino la ampliación del estudio y las intervenciones a las comunidades.

Los principales elementos de la psicología positiva son las virtudes, fortalezas y temas situacionales. Su estudio se ha vinculado en diversas investigaciones con la salud y el bienestar, encontrando aportes significativos con indicadores de salud y evaluaciones del bienestar. Sin embargo, también hay resultados con grupos particulares, en los cuales se indican que las fortalezas no están siempre asociadas con el bienestar.

Se destaca la importancia del estudio de los aspectos positivos del contexto, en donde se considera el equilibrio entre elementos positivos y negativos y el contar con conocimientos teóricos suficientes para el análisis los resultados.

Palabras clave: Bienestar, Fortalezas, Psicología Positiva, Salud, Virtudes.

\begin{abstract}
The positive psychology have had an important development in the last decades and there are efforts since the last century in the psychology field of studying positive aspects, but their development increase since 90's, that marked the beginning of the study of positive psychology.

At that time the aim it was to change the traditional point of view focused on treatment and cure to health and well-being; the aim has changed and now it points out to optimal human functioning based on virtues, character strengths, conditions and process to identify and develop the factors that contribute to thrive persons and communities. It means no only an individual approach but their study and interventions adapted to community settings.
\end{abstract}

1 Marcela Sánchez Estrada: Instituto Nacional de Salud Pública de México. Correo electrónico: mar9977@yahoo.com. 
The principal elements of the positive psychology are virtues, character strengths and situational themes, and they are being related in many studies to health and well-being. The results suggest that they have positive contributions to indicators of health and evaluations of well-being; however there are results in other studies with particular groups that suggest that character strengths do not have always the positive relationship with well-being.

It is important to consider the context in which the positive aspects are being studied, the balance between positive and negative elements, and enough theoretic knowledge for analyzing the results.

Key words: Character strengths, health, Positive psychology, virtues, well-being.

Recibido: 26 de noviembre de 2011

Aceptado: 22 de diciembre de 2011

\section{INTRODUCCIÓN}

La psicología positiva ha tenido un amplio desarrollodesdesusiniciosenlosaños9ocon su propuesta de estudiar las características positivas de las personas en condiciones normales. Se planteó en ese momento el objetivo de generar un catalizador para cambiar el objeto tradicional de reparación de los aspectos de la vida por el fomento de cualidades positivas y el bienestar (Seligman y Csikszentmihalyi, 2000).

El incremento de artículos, capítulos de libros y manuales ha sido importante en los últimos años y el interés por el área también ha sido creciente, pero se ha identificado que hay una generalización de los términos que no corresponden a la adecuada aplicación del marco teórico y que este auge notorio no necesariamente va acompañado del suficiente conocimiento teórico que permita reconocer las aportaciones y limitaciones de la psicología positiva como un área actualmente en desarrollo (Costa y López, 2008; López-Cepero, Fernández y Senin, 2009; McNulty y Fincham, 2011).

Ante estas circunstancias, es importante identificar los elementos básicos de la psicología positiva, así como algunas de sus contribuciones al bienestar, para analizar sus aportaciones a la psicología y reconocer sus áreas de oportunidad.

\section{Psicología positiva}

La iniciativa del estudio de los aspectos positivos no surge por primera vez con la psicología positiva (Froh, 2004; Linley, Joseph, Harrington y Wood, 2006), pues diversos autores los habían destacado previamente: Maslow (1954) propuso que el estudio debía basarse en el crecimiento saludable de las personas y tener como objetivo, el estudio empírico de las características de las personas autoactualizadas (Moss, 2001). María Jahoda (1958) centró sus estudios en la salud mental positiva del individuo con múltiples criterios (Lluch, 2002). Estos antecedentes han sido esfuerzos por incorporar al esquema tradicional los aspectos positivos, pero no es hasta las últimas décadas cuando se ha ampliado su estudio.

La psicología positiva se basa en estudios empíricos y en el método científico para estudiar y comprender los fenómenos psicológicos considerados positivos: las experiencias subjetivas individuales (en el pasado, presente y futuro), las característicaspersonalesylasinstituciones, 
que promueven tantolas experiencias como las características (Peterson y Seligman 2004; Seligman y Csikszentmihalyi, 2000). Es el estudio científico del funcionamiento humano a partir de fortalezas y virtudes, lo que permite identificar y promover los factores de prosperidad de las personas y comunidades. Ello implica no solo un enfoque individual, sino la ampliación del estudio y las intervenciones a las comunidades, lo cual no significa que ignora o niega los aspectos negativos; al contrario, lo que propone es que los seres humanos experimentan situaciones positivas $y$ negativas y que existe un balance vital entre las debilidades, las fortalezas y el ambiente (Aspinwall y Staudinger, 2002; Peterson y Seligman, 2004; Ironson y Powell, 2005). Las personas acentúan lo positivo en la vida al enfrentarse con situaciones negativas, y esto no es ignorar los aspectos negativos reales.

Ante la necesidad de establecer un vocabulario común para el estudio de los aspectos positivos, Peterson y Seligman (2004) han desarrollado junto con un grupo de colaboradores, un esquema de clasificación que facilite dicho estudio. Retomaron la propuesta de clasificación del Manual Diagnóstico y Estadístico de los Trastornos Mentales (DSM), para elaborar un manual de psicología positiva que incluyera virtudes, fortalezas y talentos y el estudio y evaluación de los aspectos positivos, con una postura vertical que permite especificar diferentes niveles conceptuales como parte de una jerarquía.

Desde la psicología positiva se proponen tres niveles conceptuales: Virtudes, fortalezas y temas situacionales.

Las virtudes son las características principales, pues han sido consideradas valiosas por filósofos y pensadores religiosos o espirituales. Seis son las virtudes que se han establecido como universales para esta clasificación: Sabiduría, valor, humanidad, justicia, moderación y trascendencia (Park, Peterson y Seligman, 2004).

Las fortalezas del carácter son los aspectos psicológicos de las virtudes, considerados procesos o mecanismos que definen a las virtudes. Son los caminos por los cuales se expresa una u otra virtud. Una virtud puede manifestarse por medio de diversas fortalezas; todasellasincluyenlaadquisición y uso de conocimiento, pero son diferentes entre sí. En esta clasificación se incluyen 24 fortalezas, que se distribuyen en familias por virtudes (Peterson y Seligman, 2004). Estas fortalezas favorecen el crecimiento humano y constituyen el principio fundamental de la condición humana; algunas de ellas han sido consideradas como rasgos y se han medido en diferentes etapas de la vida para identificar su desarrollo a lo largo de la misma (Seligman y Csikszentmihalyi, 2000).

Los temas o aspectos situacionales son las conductas específicas que las personas exhiben ante una situación particular con respecto a una fortaleza. Pueden ser diferentes en las culturas, géneros, cohortes y otras variables sociales importantes, además de estar vinculados a situaciones particulares (Peterson y Seligman, 2004).

Con esta propuesta se sugiere que pueden variar la expresión de las fortalezas y los temas situacionales, pero no las virtudes. Las fortalezas son el punto intermedio entre lo abstracto (virtudes) y lo concreto (aspecto situacional). De acuerdo con esta propuesta, la agrupación de las virtudes y fortalezas se muestra en la Tabla 1. 
Tabla 1. Clasificación de virtudes y fortalezas propuesta por Peterson y Seligman

\begin{tabular}{|c|c|c|c|c|c|c|}
\hline $\begin{array}{l}\mathrm{V} \\
\mathrm{I} \\
\mathrm{R} \\
\mathrm{T} \\
\mathrm{U} \\
\mathrm{D}\end{array}$ & $\begin{array}{l}\text { 1. Sabiduría y } \\
\text { conocimiento }\end{array}$ & $\begin{array}{l}2 . \\
\text { Determinación }\end{array}$ & $\begin{array}{l}3 . \\
\text { Humanidad }\end{array}$ & 4. Justicia & 5. Mesura & $\begin{array}{l}6 . \\
\text { Trascendencia }\end{array}$ \\
\hline \multirow{5}{*}{$\begin{array}{c}\mathrm{F} \\
\mathrm{O} \\
\mathrm{R} \\
\mathrm{T} \\
\mathrm{A} \\
\mathrm{L} \\
\mathrm{E} \\
\mathrm{Z} \\
\mathrm{A} \\
\mathrm{S}\end{array}$} & Creatividad & Valentía & Amor & $\begin{array}{l}\text { Pertenencia } \\
\text { a un grupo }\end{array}$ & $\begin{array}{l}\text { Perdón y } \\
\text { misericordia }\end{array}$ & $\begin{array}{l}\text { Apreciación } \\
\text { de la belleza y } \\
\text { excelencia }\end{array}$ \\
\hline & Curiosidad & Persistencia & Bondad & Igualdad & Humildad & Gratitud \\
\hline & $\begin{array}{l}\text { Apertura } \\
\text { mental }\end{array}$ & Integridad & \multirow{3}{*}{$\begin{array}{l}\text { Inteligencia } \\
\text { social }\end{array}$} & \multirow{3}{*}{ Liderazgo } & Prudencia & Esperanza \\
\hline & $\begin{array}{l}\text { Amor por } \\
\text { aprender }\end{array}$ & & & & \multirow{2}{*}{ Autorregulación } & Humor \\
\hline & Perspectiva & vicantua & & & & Espiritualidad \\
\hline
\end{tabular}

Fuente: Hope [Optimism, Future-Mindedness, Future Orientation] de Peterson y Seligman, Character Strengths and virtues. A handbook and classification (2004). Adaptado.

Uno de los resultados de este planteamiento ha sido el análisis de la propuesta tradicional de estudio de la salud y una crítica a la consideración de que esta y la enfermedad son opuestos del mismo continuo. Durante mucho tiempo se han mantenido los siguientes ejes de estudio en salud: las patologías, sus síntomas, prevalencia, costos y factores de riesgo asociados, sin dejar de lado, por supuesto, los métodos de diagnóstico y tratamiento. Sin embargo, el estudio del malestar es muy diferente del de bienestar, y la ausencia de patología o la reducción del malestar, no necesariamente implica el incremento en el bienestar per se (Vázquez y Hervás, 2009; Burns, 2010).

Es por ello que a la evaluación de la salud se ha incorporado la del bienestar con dos principales propuestas: el bienestar subjetivo y el bienestar psicológico. El primero hace referencia a la evaluación que las personas hacen de su vida, la cual puede comprender juicios sobre la satisfacción y evaluación de los estados emocionales placenteros y displacenteros, y por esta razón tiene dos componentes: el afectivo, que es el balance entre el placer y el displacery el cognoscitivo, que corresponde al juicio sobre la satisfacción con la vida (Diener y Lucas, 1999; Reig y Garduño, 2005). El segundo, bienestar psicológico desarrollado por Ryff, incluye un esquema de seis dimensiones: la autoaceptación, el crecimiento personal, la autonomía, el propósito en la vida, el dominio del control del ambiente y las relaciones positivas con los otros, consideradas como índices del bienestar y que integran distintos aspectos del funcionamiento humano óptimo (Ryff, 1989; Ryff y Keyes, 1995; Ryff y Singer, 2003).

Con la inclusión del bienestar en la evaluación de la salud, se ha identificado que aquel es un factor importante en la mortalidad por diferentes causas (accidentes, enfermedad cardiovascular) y que en niveles bajos es un factor de riesgo. En el caso específico del componente cognitivo, se ha sugerido como un potencial predictor de quienes tienen riesgos para problemas de salud (Love, Glei y Goldman, 
2009). También para las enfermedades cardiovasculares, los niveles bajos se han asociado con dolor crónico e infecciones respiratorias, así como con episodios de depresión (Frisch et al., 2003).

Esta es la razón para que su estudio se haya ampliado recientemente en diversas disciplinas, con el objetivo de conocer qué hacen las personas para estar bien, disfrutar de la vida y tener un nivel óptimo de desarrollo en las diferentes áreas de ella. El bienestar se ha vinculado a diversos indicadoresy objetivos económicos, de salud y sociales, pero en los últimos años se han incluido positivos aspectos subjetivos que han sido considerados cada vez más para su estudio a nivel nacional e internacional (Millán, 2011; Rojas, 2011).

Diversos autores han propuesto que el malestar y bienestar son procesos relativamente independientes, ya que se ha encontrado en una variedad de estudios que la asociación entre depresión y bienestar va de -0.40 a -0.55 (Slade, 2010), mientras que para ansiedad y bienestar ha sido reportada de -0.53 y -0.63. Esto sugiere que no son opuestos del mismo continuo, y que si bien hay cierto grado de relación, esta no es perfecta. Por lo tanto, tener como objetivo el malestar y sus factores de riesgo es justamente trabajar en este sentido y no implica necesariamente que con esto incremente el bienestar. Incluir mediciones de fortalezas a las ya tradicionales evaluaciones, podría dar un indicador de cómo abordar esta perspectiva de salud y bienestar (Elderen, Maes y Dusseldorf, 1999; Davis, 2005; Keyes, 2005; Vázquez y Hervás, 2009).

\section{Aportaciones de la psicología positiva}

Los estudios relacionados con aspectos positivos (principalmente fortalezas $y$ emociones positivas), se han asociado con menos días de hospitalización y reducción de índices de mortalidad por problemas cardiacos e hipertensión. Las emociones positivas se han identificado como predictoras de un mejor autorreporte de salud y disminución de síntomas y dolor (Ardelt, 2003; Cohen y Pressman, 2006; Eun-Kyoung, 2007; Fernández y Ballesteros, 2009). En otros casos dan una mejor respuesta a tratamientos médicos (Mueller, Plevak y Rummans, 2001; Snyder, Sympson, Michel y Cheavens, 2002; Powell, Shahabi y Thoresen, 2003; Lee y Newberg, 2005). Otros muestran una asociación positiva con el bienestar (Park, Peterson y Seligman, 2004; Davis, 2005; Sawatzky, Ratner y Chiu, 2005). Algunos señalan aumento en ciertos procesos como la intuición, la creatividad $y$ el funcionamiento cognitivo (Bailly $y$ Roussiau, 2010).

Collins, Goldman y Rodríguez (2008) analizaron la relación entre el bienestar y la salud. Varios estudios sugieren que el afecto positivo es un factor protector para el desarrollo de alteraciones de movilidad y para la mortalidad, y se ha encontrado una asociación negativa con niveles de depresión, ansiedad y estrés (EunKyoung, 2007; Sin y Lyubomirsky, 2009).

Ante los hallazgos con diferentes poblaciones, parecería que la psicología positiva es un área de suma importancia, y en muchos casos se ha considerado como el camino más certero para trabajar en pro de la salud y el bienestar. Sin embargo, también hay resultados que sugieren ser cautelosos con esta aseveración. McNulty y Fincham (2011) han propuesto que las fortalezas por símismas no traen consigo el bienestar y que es fundamental considera el contexto en el cual se expresan estas. Además, han reportado que algunas fortalezas no tienen siempre un efecto 
positivo en el bienestar, por ejemplo, en grupos de mujeres cuyas parejas presentan comportamientos hostiles y en jugadores compulsivos. Fortalezas como el perdón y el optimismo no se han asociado a largo plazo con un incremento en su bienestar, ya que se mantienen en contextos que afectan su integridad y que pueden volverse peligrosos.

\section{CONCLUSIONES}

La psicología positiva ha facilitado el estudio de aspectos a los cuales se les había prestado poca atención, como las experiencias subjetivas y las emociones positivas y ha permitido destacar la salud y el bienestar sobre el modelo tradicional de curación. Sin embargo, no es posible considerar solo estos aspectos positivos para estudiar el bienestar. Es necesario identificar sus características, estudiarlos $y$ analizarlos en el contexto, ya que es el balance entre los aspectos negativos y positivos (en esta relación), lo que permite proponer un modelo de atención a la salud (Aspinwall y Staudinger, 2002; Peterson y Seligman, 2004).

El modelo tradicional ha contribuido a ampliar el conocimiento para el tratamiento de las patologías y a reducir el malestar que afecta a diversos grupos. Retomando las experiencias en este campo, es posible reconocer las aportaciones de la psicología positiva y enfatizar la importancia de la preparación teórica, del contexto específico en el cual se estudian, de las características de la población y de la metodología para su estudio. Todos estos elementos son fundamentalesparapoderrealizarunanálisis crítico de las aportaciones y limitaciones de la psicología positiva, teniendo en cuenta que aunque se ha marcado la posibilidad de generar intervenciones desde una perspectiva de prevención, es indispensable tener el sustento teórico y empírico para poder hacerlo de tal manera que incidan efectivamente en el bienestar de las personas a largo plazo.

Diversos autores han propuesto que la psicología no es positiva ni negativa y que más que un adjetivo que posicione una por encima de otra, la psicología es una y tiene como objetivo estudiar y mejorar la vida de las personas y las condiciones de las instituciones y de las comunidades (Costa y López, 2008; McNulty y Fincham, 2011). De tal forma, el estudio de los aspectos positivos es fundamental, partiendo de los hallazgos en grupos sanos (es decir, que inicialmente no tienen afectaciones en su funcionamiento cotidiano) $y$ extendiendo tal estudio a grupos con características particulares para identificar el comportamiento de los aspectos positivos en ellos y las diferencias en el diseño de intervenciones adecuadas a sus necesidades y características. En este sentido, es importante reconocer estas diferencias para retomar en su justa dimensión, los resultados y el alcance de los mismos, lo cual permitirá incrementar el conocimiento de estos fenómenos y realizar intervenciones efectivas.

\section{REFERENCIAS}

Ardelt, M. (2003). Effects of Religion and Purpose in Life on Elders' Subjective Well-Being and Attitudes toward Death. Journal of Religious Gerontology, 14(4), 55-77. Recuperado de http://www.clas. ufl.edu/users/ardelt/Effects $\% 200$ f 20 Religion\%20and\%20Purpose\%20in\%20 Life.pdf.

Aspinwall, L. G. y Staudinger, U. M. (2002). A psychology of Human Strengths: Some. Central Issues of an Emerging Fields. En L. G. Aspinwall y U. M. A. 
Staudinger (Eds.), Psychology of Human Strengths. Fundamental Questions and Future Directions for a Positive Psychology (pp. 9-22). Washington, D. C, EE. UU.: American Psychological Association.

Bailly, N. y Roussiau N. (2010). The Daily Spiritual Experience Scale (DSES): Validation of the Short Form in an Elderly French Population. Canadian Journal of Aging, 29 (2), 223-231. doi:10.1017| So714980810000152.

Burns, G. W. (2010). Now I Can Get on with Life. Pleasure, Engagement, and Meaning in a Case of Major Depression. En G. W. Burns (Ed.), Happiness, Healing, Enhancement. your Casebook Collection for Applying Positive Psychology in Therapy (pp. 127-138). Hobokenn NJ, EE. UU.: John Wiley.

Cohen, S. y Pressman, S. D. (2006). Positive Affect and Health. Current Directions in Psychological Science, 15(3), 122-125. doi:10.1111/j.0963-7214.2006.00420.x.

Collins, A. L., Goldman, N. y Rodríguez, G. (2008). Is Positive well-being protective of mobility limitations among older adults? Journal of Gerontology: Psychological Sciences, 63, 321-327. Oxford, Inglaterra.

Costa, M. y López, E. (2008). La perspectiva de la potenciación en la intervención psicológica ( $\mathrm{a}^{\mathrm{a}}$ ed.). En C. Vázquez, y G. Hervás. (Eds.), Psicología positiva aplicada, (pp. 75-100). Sevilla, España: Desclee de Brouwer.

Davis, B. (2005). Mediators of the Relationship between Hope and Wellbeing in Older Adults. Clinical Nursing Research, 14(3), 253-272. University of Pennsylvania, School of Nursing, USA.
Diener, E. y Lucas, E. R. (1999). Personality and Subjective Well-being. En D. Kahneman, E. Diener y N. Schwartz, (Eds.). Well-being: The Foundations of Hedonic Psychology (pp.150-200). New York, NY, EE. UU.: Russell Sage Foundation.

Elderen, T., Maes, S. y Dusseldorf, E. (1999). Coping with Coronary Heart Disease: A Longitudinal Study. Journal of Psychosomatic Research, 47 (2), 175183. PMID: 10579500.

Eun-Kyoung, O. L. (2007). Religion and Spirituality as Predictors of Well-Being among Chinese American And Korean American Older Adults. Journal of Religious, Spirituality y Aging, 19 (3), 77100. doi: 10.1300/J496v19no3_06.

Fernández-Ballesteros, R. (2009). El bienestar de los mayores. En C. Vázquez, y G. Hervás (Eds.), Psicología positiva aplicada. (pp.371401). Sevilla, España: Desclee de Brouwer.

Frisch, M. B., Clark, M. P., Rouse, S. V., Rudd, M. D., Paweleck, J., Greenstone, A. y Kopplin, D. A. (2003). Predictive Validity and Sensitivity to Change in Quality of Life Assessment and Life Satisfaction: Further Studies of the Quality of Life Inventory or QOLI in Mental Health Settings. En M. J. Sirgy, D. Rahtz y A. C. Samli (Eds.), Advances in Quality of Life: Theory and Research. (pp. 191210). Greenstone, Netherlands: Kluwer Academic Publishers.

Froh, J. J. (2004). The History of Positive Psychology: Truth Be Told. NYS Psychologist, May-June, 18-20. Recuperado de http://people.hofstra. edu/jeffrey_j_froh/Froh,J.J._The\%20 History\%200f\%20Pos\%20Psyh(2004).pdf. 
Henry J. (2007). Positive Psychology and the Development of Well-being. In J. Haworth, G. Hart (Eds.), Well-being: Individual, Community And Societal Perspectives (pp. 25-40). Basingstoke. Recuperado de http://oro.open. ac.uk $/ 27745 /$.

Ironson, G. H., y Powell, L. H. (2005). An Exploration of the Health Benefits of Factors that Help us to Thrive. International Journal of Behavioral Medicine. 12(2), 47-49. PMID: 15901212.

Jahoda, M. (1958). Current Concepts of Positive Mental Health. New York, NY, EE. UU.: Basic Books.

Keyes, C. L. M. (2005). Mental Illness and/or Mental Health? Investigating Axioms of the Complete State Model of Health. Journal of Consulting and Clinical Psychology, 73(3), 539-548. Recuperado de http://textedu.com/f2/download/ mental-illness-and-or-mental-healthinvestigating-axioms-of-the-completestate-model-of-health.pdf.

Lee, B.Y. y Newberg, A. B. (2005). Religion and Health: A Review and Critical Analysis. Zygon, 40 (2), 443-468. doi: 10.1111/j.1467-9744.2005.00674.x.

Linley, P. A., Joseph, S., Harrington, S. y Wood, A. M. (2006). Positive Psychology: Past, Present, and (possible) Future. The Journal of Positive Psychology, 1(1), 3-16. doi: 10.1080/17439760500372796.

Lluch, M. T. (2002). Evaluación empírica de un modelo conceptual de salud mental positiva. Salud Mental, 25(4), 42-55. Recuperado de http://www. medigraphic.com/pdfs/salmen/sam2002/sam024e.pdf.
López-Cepero, J. B., Fernández, J. E. y Senin, C. C. (2009). Ten Major Reviews about Positive Psychology. Annuary of Clinical and Health Psychology, 5, 4753. Recuperado de http://institucional. us.es/apcs/doc/APCS_5_eng_47-53.pdf.

Love, C. A., Glei, D. A. y Goldman, N. (2009). The Role of Life Satisfaction and Depressive Symptomsinall-CauseMortality.Psychology Aging, 24(3), 696-702.

Maslow, A. H. (1954). Motivation and Personality. NewYork, NY, EE. UU.: Harper y Row Publishers.

McNulty, J. K., y Fincham, F. D. (2011). Beyond Positive Psychology? : Toward a Contextual View of Psychological Processes and Well-Being. American Psychologist. doi: 10.1037/a0024572.

Millán, R. (2011). El bienestar como el nuevo “objeto" del progreso. Cinco reflexiones. En J. P. Laclette (Coordinador general), La medición del progreso y del bienestar. Propuesta desde América Latina. (pp. 19-28). Ciudad de México, México: Foro Consultivo Científico y Tecnológico.

Moss, D. (2001). The Roots and Genealogy of Humanistic Psychology. In K. J. Schneider, J. F. T. Bugental y J. F. Pierson (Eds.). The Handbook of Humanistic Psychology: Leading Edges in Theory, Research and Practice (pp. 5-20). Thousand Oaks, CA, EE. UU.: Sage Publications.

Mueller, P.S., Plevak, D.J. y Rummans. T. A. (2001). Religious Involvement, Spirituality and Medicine: Implications for Clinical Practice. Clinic Procedure, 76 (12), 1225-1235. Rochester, Minnesota, EE. UU. 
Park, N., Peterson, C. y Seligman, E. P. M. (2004). Strengths of Character and Well-being. Journal of Social and Clinical Psychology, 23(5), 603-619. doi: 10.1521/ jscp.23.5.603.50748.

Peterson, C., y Seligman, E. P. (2004). Introduction to a "Manual of the Sanities". Character Strengths and Virtues. A Handbook and Classification. Washington, D. C., EE. UU.: American Psychological Association.

Powell, L. H., Shahabi, L. y Thoresen, C. E. (2003). Religion and Spirituality. Linkages to Physical Health. American Psychologist, 58(1), 36-52. Chicago, IL, EE. UU. doi: 10.1037/0003-066X.58.1.36.

Reig, P., E. y Garduño, E. L. (2005). Procesos cognoscitivos asociados a los constructos de calidad de vida y bienestar subjetivo. En E. L. Garduño, A. B. Salinas y H. M. Rojas (Eds.), Calidad de vida y bienestar subjetivo en México (pp.57-82). Ciudad de México, México: Plaza y Valdés Editores.

Rojas, M. (2011). Más allá del ingreso: progreso y bienestar subjetivo. En J. P. Laclette (Coordinador general), La medición del progreso y del bienestar. Propuesta desde América Latina (pp.2940). Ciudad de México, México: Foro Consultivo Científico y Tecnológico.

Ryff, C. D. (1989). Happiness Is Everything, or is it? Explorations on the Meaning of Psychological Well-Being. Journal of Personality and Social Psychology, 57(6), 1069-1081. doi: 10.1037/00223514.57.6.1069.

Ryff, C. D. y Keyes, C. L. (1995). The Structure of Psychological Well-Being (Revised). Journal of Personality and
Social Psychology, 69(4), 719-727. doi: 10.1037/0022-3514.69.4.719.

Ryff, C. D. y Singer, B. (2003). Ironies of the Human Condition: Well-Being and Health on the Way to Mortality. En L. G. Aspinwall, y U. M. Staudinger (Eds.), A Psychology of Human Strengths. Fundamental Questions and Future Directions for a Positive Psychology (pp. 271-288). San Diego, CA, EE. UU.: Academic Press.

Sawatzky, R., Ratner, P. A. y Chiu, L. (2005). A Meta-Analysis of the Relationship between Spirituality and Quality Of Life. Social Indicators Research, 72 (2), 153188. doi: 10.1007/s11205-004-5577-x.

Seligman, M. P. y Csikszentmihalyi, M. (2000). Positive Psychology: An Introduction. American Psychologist, 55(1), 5-14. doi: 10.1037/0003-066X.55.1.5.

Seligman, M. E. y Peterson, C. (2002). Positive Clinical Psychology. En L. G. Aspinwall Y U. M. A. Staudinger (Eds.), Psychology of Human Strengths. Fundamental Questions and Future Directions for a Positive Psychology (pp. 305-318). Washington, D. C, EE. UU: American Psychological Association.

Sin, N. L.y Lyubomirsky, S. (2009). Enhancing Well-Being and Alleviating Depressive Symptoms with Positive Psychology Interventions: A Practice-Friendly MetaAnalysis. Journal of Clinical Psychology, 65(5), 467-487. doi: 10.1002/jclp.20593.

Slade, M. (2010). Mental Illness and Wellbeing:The Central Importance of Positive Psychology and Recovery Approaches. BMC Health Services Research, 10(26), 2-14. doi:10.1186/1472-6963-10-26. 